\title{
Renal p38 MAP kinase activity in experimental diabetes
}

\author{
Radko Komers ${ }^{1,2}$, Jessie N Lindsley ${ }^{1}$, Terry T Oyama ${ }^{1}$, David M Cohen ${ }^{1,3}$ and Sharon Anderson ${ }^{1,3}$
}

Renal cell activity of p38 mitogen-activated protein kinase (p38) is increased in the diabetic milieu. p38 mediates signals relevant for the development of diabetic nephropathy (DN). However, renal p38 in Type 1 diabetes in vivo, particularly in conditions reflecting the differences in metabolic control, and its activity in advanced stages of DN, has received less attention. We examined the p38 pathway in renal cortex of rats with streptozotocin diabetes (4 weeks) with poor (DS), moderate (DM), and intensive (DII) metabolic control, achieved by varying doses of insulin therapy. Renal p38 was also studied in 12-month diabetic rats with established nephropathy (DM12) and compared with age-matched controls. p38 activity (in vitro kinase assay and expression of phosphorylated (active) p38 (P-p38)) was increased in DM and DS rats, as compared with non-diabetic controls, and attenuated by intensive insulin treatment. In all groups, P-p38 was predominantly localized in macula densa cells. Diabetic rats also demonstrated P-p38 immunoreactivity in the distal tubule and glomeruli. Enhanced p38 activity in DS and DM rats was not associated with increases in expression of active mitogen-activated protein kinase $3 / 6$, an activator of p38, but paralleled with increased expression of scaffolding protein transforming growth factor- $\beta$-activated protein kinase 1-binding protein 1. Expression of mitogen-activated protein phosphatase-1 (MKP-1), one of the phosphatases involved in inactivation of mitogen-activated protein kinase signaling, was increased in all diabetic groups, irrespective of metabolic control. Renal p38 activation was also detectable in D12 rats with established albuminuria and glomerulosclerosis. In summary, renal cortical p38 activity was increased in diabetic rats at early and advanced stages of nephropathy, as compared with non-diabetic animals, and attenuated by improved metabolic control. p38 activation in diabetes is likely to occur via multiple pathways and cannot be explained by downregulation of MKP-1.

Laboratory Investigation (2007) 87, 548-558; doi:10.1038/labinvest.3700549; published online 2 April 2007

KEYWORDS: diabetic nephropathy; hyperglycemia; p38 mitogen-activated protein kinase; proteinuria; signaling

High glucose concentrations, and potentially other factors in the diabetic milieu, trigger pathophysiological signals mediated by the activation of specific kinase cascades. Altered activation of mitogen-activated protein (MAP) kinases (MAPK) occurs under these conditions and results in a broad spectrum of acute and long-term effects including mobilization of intracellular calcium and induction of gene expression. ${ }^{1}$ In the kidney, such aberrant activation of MAPK cascades may perturb the balance of humoral and cytokine systems responsible for regulating vascular tone, permeability, renal cellular growth, and composition of the extracellular/mesangial matrix (ie, factors associated with the development of diabetic nephropathy). ${ }^{2,3}$

p38 mitogen-activated protein kinase (p38), a member of the MAPK family, is activated by a number of stimuli. As a stress-activated kinase, p38 is activated by physical and chemical stress factors, inflammatory cytokines, and vasoactive and growth factors, resulting in growth promotion, apoptosis, oxidative stress, and vasoconstriction. ${ }^{4-7}$ In the MAPK signaling cascade, p38 is typically activated by MAP kinase kinase 3/6 (MKK 3/6) and translocated to nuclei. Active (phosphorylated) p38 then, in turn, phosphorylates and activates nuclear effectors such as the transcription factors activating transcription factor-2 (ATF-2) and cyclic AMP response element binding protein (CREB). ${ }^{4,5}$ More recently, mitogen-activated protein kinase 3/6 (MKK3/6) independent mechanism of p38 activation-has been described in various cell types. This mechanism involves interaction between p38 and transforming growth factor$\beta$-activated protein kinase 1-binding protein 1 (TAB1), leading to autophosphorylation and activation of the kinase. $^{8}$

\footnotetext{
${ }^{1}$ Division of Nephrology and Hypertension, Oregon Health and Science University, Portland, OR, USA; ${ }^{2}$ Diabetes Center, Institute for Clinical and Experimental Medicine, Prague, Czech Republic and ${ }^{3}$ Medical Service, Portland VA Medical Center, Portland, OR, USA

Correspondence: Dr R Komers, MD, PhD, Division of Nephrology and Hypertension, Oregon Health and Science University, PP262, 3314 SW US Veterans Hospital Road, Portland, OR 97239-2940, USA. E-mail: komersr@ohsu.edu
} 
Inactivation of MAPK signaling, including $\mathrm{p} 38$, is mediated through dephosphorylation via a class of dual specificity MAPK-directed protein phosphatases. ${ }^{9}$ Mitogen-activated protein phosphatase-1 (MKP-1), the most ubiquitously expressed and best studied of these phosphatases, has dual catalytic activity toward phosphotyrosine and phosphothreonine, and inactivates $\mathrm{p} 38$ as well as other MAPK in vitro and in vivo. ${ }^{9}$ Inducible overexpression of MKP-1 was used to demonstrate that MKP-1 preferentially inactivates p38 MAPK, and to a lesser extent other MAP kinases. ${ }^{10}$

Convincing in vitro data support a role for $\mathrm{p} 38$ in the cell response to a diabetic milieu. The p38 pathway is activated in rat aortic vascular smooth muscle cells (VSMC), ${ }^{11,12}$ in renal cells cultured in high-glucose media, ${ }^{3,13-16}$ in isolated glomeruli of streptozotocin (STZ)-diabetic rats, ${ }^{2,15}$ and is involved in mediating signals relevant for development of diabetic nephropathy.

In contrast to relatively abundant in vitro evidence suggesting renal cell p38 activation in the diabetic milieu, renal p38 signaling in Type 1 diabetes in vivo, particularly in clinically more relevant conditions reflecting to differences in metabolic control and insulin treatment, has received less attention. Moreover, it has been suggested that the time factor may be important in assessing the role of p38 in diabetic renal pathophysiology. Studies conducted in isolated glomeruli have suggested that p38 activation is a feature of early nephropathy in STZ-diabetic rats. ${ }^{2,15}$ However, in Type 2 diabetic patients with biopsy-proven nephropathy, p38 immunoreactivity localized both in glomerular and nonglomerular renal compartments, suggesting that renal cortical p38 activation could be detected even in more advanced stages of the disease. ${ }^{17,18}$ Thus, whether p38 activation could be detected in advanced stages of the disease in Type 1 diabetes remains to be elucidated.

To address these issues, we assessed activation of the p38 pathway in renal cortex of STZ-diabetic rats in early stages of nephropathy with different levels of glycemic control, achieved by varying insulin treatment. In addition, we determined renal p38 activity in diabetic rats with advanced nephropathy, associated with progressive proteinuria and glomerulosclerosis.

\section{MATERIALS AND METHODS Diabetic Rat Model}

Studies were conducted in adult male Sprague-Dawley rats, with initial weights $\sim 300 \mathrm{~g}$. The rats were made diabetic by intraperitoneal (i.p.) injection of streptozotocin (Sigma, St Louis, MO, USA), $65 \mathrm{mg} / \mathrm{kg}$ body weight. Three days later, induction of diabetes was confirmed by measurements of tail blood glucose (BG) level using a reflectance meter (One Touch II; Lifescan, Milpetas, CA, USA). The studies were approved by the Portland Veteran Affairs Institutional Animal Care and Use Subcommittee.

\section{Study Design}

Protocol 1. Renal p38 MAP kinase activity and expression in early experimental diabetes: role of metabolic control

Diabetic rats were then randomized to receive $4 \mathrm{IU}$ of ultralente insulin per day (Humulin U, Eli Lilly, Indianapolis, IN, USA) (moderately hyperglycemic diabetic rats, DM, $n=8$ ), no insulin treatment (severely diabetic rats, DS, $n=8$ ), or $12 \mathrm{IU}$ of insulin/day (diabetic rats on intensive insulin treatment, DII, $n=8$ ), to achieve near normal metabolic control. Age-matched non-diabetic Sprague-Dawley rats served as controls $(\mathrm{C}, n=8)$. All rats were fed standard rat chow (Rodent Laboratory Chow 5001, Ralston Purina, Richmond, IN, USA) ad libitum. After 3-4 weeks of diabetes, the rats were anesthetized with i.p. injection of methohexitone (Brevital, $50 \mathrm{mg} / \mathrm{kg}$ ) and blood samples for determinations of BG and glycosylated hemoglobin (HbAlc) were obtained from the abdominal aorta followed by tissue collections for protein expression studies.

\section{Protocol 2. Renal p38 activity in diabetic rats with established nephropathy}

Control (C12) and moderately hyperglycemic diabetic rats (DM12, 4 IU of ultralente insulin per day) were used in these studies. After 12 months of follow-up, the rats were placed in metabolic cages to obtain 24-h urine collections for determination of albuminuria. Several days after these measurements, the rats were killed with blood and tissue harvesting as described above.

\section{Tissue Preparation}

The kidneys were removed, decapsulated, divided into cortical and medullary portions, and snap-frozen in liquid nitrogen. Kidney cortices were homogenized in RIPA buffer containing $50 \mathrm{mM}$ Tris, $150 \mathrm{mM} \mathrm{NaCl}, 0.5 \%$ sodium deoxycholate, $0.1 \%$ SDS and $1.0 \%$ Triton-X 100, protease inhibitors (PMSF $40 \mu \mathrm{g} / \mathrm{ml}$, leupeptin $20 \mu \mathrm{g} / \mathrm{ml}$, benzamidine $20 \mu \mathrm{g} / \mathrm{ml}$ ), and phosphatase inhibitors ( $\mathrm{NaF} 1 \mathrm{mM}$, activated sodium orthovanadate $1 \mathrm{mM}$, microcystin LR $5 \mathrm{nM}$, sodium pyrophosphate $1 \mathrm{mM}$, and PNPP $1 \mathrm{mM}$ ), incubated on ice for $1 \mathrm{~h}$, centrifuged at $12000 \mathrm{~g}$ for $10 \mathrm{~min}$ at $4^{\circ} \mathrm{C}$. The resulting supernatant was stored at $-70^{\circ} \mathrm{C}$ until analysis by p38 activity assay and Western blotting.

To obtain nuclear fractions, the pellet obtained from RIPA preparation was suspended in $0.2 \mathrm{ml}$ of buffer B $(20 \mathrm{mM}$ HEPES (pH 7.9), 25\% glycerol, $1.5 \mathrm{mM} \mathrm{MgCl}_{2}, 420 \mathrm{mM}$ $\mathrm{NaCl}, 0.5 \mathrm{mM}$ DTT, $0.2 \mathrm{mM}$ EDTA, $0.5 \mathrm{mM}$ PMSF, and $4 \mu \mathrm{M}$ leupeptin) and incubated on ice for $2 \mathrm{~h}$ with intermittent mixing. The suspension was then centrifuged at 14000 r.p.m. at $4^{\circ} \mathrm{C}$ for $30 \mathrm{~min}$. The supernatant (nuclear extract) was collected and stored at $-70^{\circ} \mathrm{C}$ until use. Total protein content in fractions was determined by BCA analysis (Pierce Biotechnology, Rockford, IL, USA). To confirm the validity of the process of nuclear fractionation, protein expression of endogenous phosphorylated ATF-2 in whole-cell nuclei-free and nuclear fractions was determined by Western blotting 
(see below). These experiments demonstrated substantial enrichment of nuclear fractions with the phospho-ATF-2 (Figure 3b).

\section{Determination of p38 MAPK Activity Using the ATF-2 Assay}

This procedure was performed using a commercially available kit according to the manufacturer's recommendations (Cell Signaling, Beverly, MA, USA). In brief, renal cortical nucleifree homogenates $(300 \mu \mathrm{g})$ were immunoprecipitated with immobilized anti-phospho-p38 antibody and incubated with the p38 substrate ATF-2 in the presence of ATP. Protein expression of phosphorylated ATF-2, as a measure of p38 activity, was then determined by immunoblotting using a specific antibody against phospho-ATF-2 (Thr 69/71).

\section{Immunoblotting}

Denatured proteins were separated on an SDS-polyacrylamide gel and transferred to PVDF membranes (BioRad) and blocked with TBS-T containing 5\% non-fat dry milk. Following blocking, membranes were incubated overnight with rabbit anti-phospho-p38 (Thr180/Tyr182) or antiphospho-MKK3/6 (Ser189/207) primary antibodies (Cell Signaling, 1:500). Immunodetection was accomplished using goat anti-rabbit secondary antibody conjugated with horseradish peroxidase (HRP) for $60 \mathrm{~min}(1: 80000$, Pierce) in TBS-T containing $5 \%$ non-fat dry milk. Visualization was performed with an enhanced chemiluminiscence (ECL) Western blotting kit (Supersignal West Dura, Pierce). Following the detection of phospho-proteins, the membranes were stripped and reincubated with rabbit polyclonal antibodies against total p38 and MKK3 (Cell Signaling, 1:1000), and further processed as described above. To confirm equality of loading, membranes were restripped and reanalyzed for actin expression (Santa Cruz Biotechnology, Santa Cruz, CA, USA).

Measurements of TAB1 and MKP-1 expression were accomplished using the same procedure as described above using rabbit polyclonal antibodies, (1:500, Santa Cruz, Alexis) followed directly by actin detection. Resultant films (Kodak) were scanned using a flatbed scanner and images analyzed with NIH Image software with the gel plotting macro. All kinase assays and Western blot analyses were performed at least in quadruplicate.

\section{Immunohistochemistry}

The halves of excised left kidneys were immersed in 10\% formalin. The fixed kidneys were dehydrated through a graded series of ethanols, embedded in paraffin, sectioned at $4 \mu \mathrm{m}$ thickness, and placed onto glass slides. The immunohistochemical procedure was performed as previously described $^{19}$ using primary antibodies against phospho-p38 (Cell Signaling, 1:200). In control and diabetic rats undergoing the long-term follow-up, the kidney paraffin sections were also stained with periodic acid Schiff (PAS)-stained sections for the assessment of glomerular morphology.

\section{Analytical Methods}

HBA1c was detected by affinity column chromatography (Glyco-Gel B, Pierce). Urinary albumin concentrations were determined using the Nephrat kit (Exocell Inc., Philadelphia, PA, USA).

\section{Statistical Analysis}

Data are expressed as means \pm s.e.m. Inter-group analyses were performed by ANOVA factorial. Statistical significance was defined as a $P$-value $<0.05$.

\section{RESULTS}

\section{Renal p38 MAP Kinase Activity in Early Experimental Diabetes: Role of Metabolic Control}

General characteristics in control and diabetic rats are presented in Table 1. DS rats demonstrated reduced weight gain, as compared with control animals and other groups of diabetic rats. Furthermore, body weights in DII rats were greater than in DM rats. All groups of diabetic rats demonstrated renal hypertrophy assessed both by kidney weight and kidney to body weight ratios, and significant increases in blood glucose concentration and HBA1c. In diabetic rats, both parameters of metabolic control reflected well the differences in insulin treatment. The highest BG and HBAlc levels were observed in rats with no insulin treatment. There were sig-

Table 1 General characteristics of control and diabetic rats

\begin{tabular}{|c|c|c|c|c|c|c|}
\hline Group & $n$ & BWT (g) & LKW (g) & LKW/BWT & BG (mg/dl) & HBA1c (\%) \\
\hline C & 8 & $353 \pm 3$ & $1.14 \pm 0.06$ & $0.33 \pm 0.02$ & $83 \pm 8$ & $4.6 \pm 0.5$ \\
\hline DS & 8 & $284 \pm 16^{\mathrm{b}, \mathrm{d}, \mathrm{e}}$ & $1.68 \pm 0.16^{b}$ & $0.59 \pm 0.04^{b, d, f}$ & $342 \pm 13^{\mathrm{b}, \mathrm{d}, \mathrm{e}}$ & $14.9 \pm 0.9^{\mathrm{b}, \mathrm{d}, \mathrm{e}}$ \\
\hline DII & 8 & $376 \pm 7^{c}$ & $1.81 \pm 0.02^{b}$ & $0.48 \pm 0.01^{b}$ & $172 \pm 10^{\mathrm{b}}$ & $7.9 \pm 0.5^{\mathrm{a}}$ \\
\hline
\end{tabular}

BWT, body weight; LKW, left kidney weight; BG, blood glucose; HBA1C, glycosylated hemoglobin; C, control; DM, moderately hyperglycemic diabetic rats; DS, severely diabetic rats, no insulin treatment; DIl, diabetic rats on intensive insulin treatment.

${ }^{\mathrm{a}} P<0.05$ vs control; ${ }^{\mathrm{b}} P<0.01$ vs control; ${ }^{\mathrm{c}} P<0.05$ vs DM; ${ }^{\mathrm{d}} P<0.01$ vs DM; ${ }^{\mathrm{e}} P<0.01$ vs $\mathrm{DIl} ;{ }^{\mathrm{f}} P<0.05$ vs DII. 
nificant differences in BG and HBAlc among the groups of diabetic rats, consistent with intensity of insulin treatment.

\section{Renal Cortical p38 MAPK Activity and Expression}

Analysis of renal cortical p38 activity was first determined by in vitro kinase assay (Figure 1). Using this approach, p38 kinase activity was found to be increased in DM and DS, as compared with controls. In DII, p38 activity was not different from controls. Activation of p38 kinase was also quantitated by measurements of expression of phosphorylated (active) p38 (P-p38) and P-p38/total p38 protein ratios (Figure 2). These studies revealed significant increases in DS rats, lacking insulin treatment. Similar to DS rats, moderately hyperglycemic diabetic rats demonstrated increases in all parameters characterizing p38 activity in nuclei-free whole-cell samples compared with control animals. However, the P-p38 expression in DM rats was lower as compared with DS rats (Figure 2). Although numerically comparable to DM rats, Pp38 expression in DII rats was not statistically different from controls. However, the P-p38/total p38 remained elevated in these rats even despite intensive insulin treatment (Figure 2). Total p38, consisting of both active and inactive kinase, did not differ between groups.

Following activation, phosphorylated p38 translocates to the nucleus, and interacts with and phosphorylates downstream effectors. ${ }^{20}$ Since the nuclear P-p38 represents the most important pool of the kinase with respect to its roles in a variety of biological processes, further analyses determined nuclear expression of $\mathrm{p} 38$. Consistent with data using wholecell lysates, nuclear expression of P-p38 was increased in DM and DS rats, but not in DII rats, as compared with control

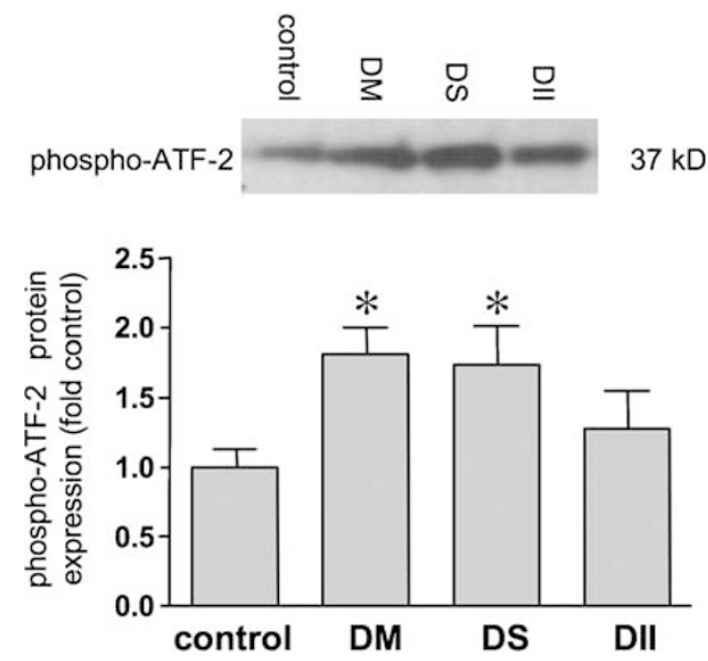

Figure 1 Renal cortical p38 MAPK activity in control and diabetic rats. Renal cortical p38 activity, determined by in vitro kinase assay (whole-cell nuclei-free fractions) using ATF-2 as a substrate, was increased in moderately (DM) and severely diabetic (DS) rats. In diabetic rats treated with intensive insulin regimen (DII), the p38 MAPK activity was not different from controls. A representative blot of phospho-ATF- 2 is shown in the upper panel. ${ }^{*} P<0.05$ vs control. animals (Figure 3). There were no differences in total nuclear p38 between the groups.

In all groups of rats, phospho-p38 was predominantly localized in macula densa cells (Figure 4). Unlike the control animals, all groups of diabetic rats also demonstrated phospho-p38 immunoreactivity in the distal tubules. Glomerular

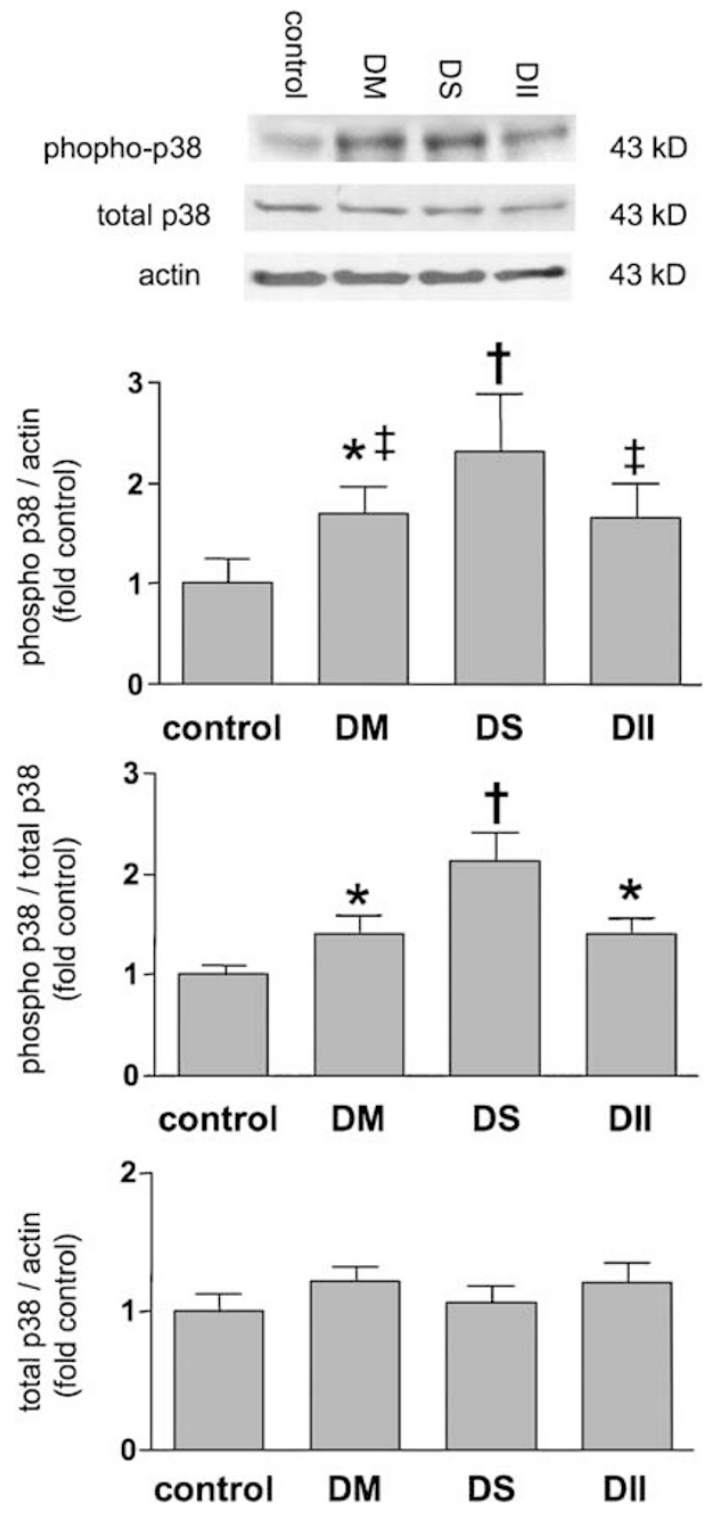

Figure 2 Renal cortical expression of phosphorylated (P-p38) and total p38 MAPK in whole cell nuclei-free fractions. Western blot analysis was used to determine $\mathrm{p} 38$ expressions, as described in Materials and methods. P-p38 expression (P-p38/actin) and P-p38/total p38 protein ratios were significantly increased in moderately diabetic rats (DM), and in rats with severe diabetes (DS), as compared with control animals. However, the Pp38 expression in DM rats was lower than in DS rats. P-p38 expression in DII rats was not statistically different from controls, and significantly reduced as compared with DS, whereas the P-p38/total p38 ratio remained elevated in these rats even despite intensive insulin treatment. Total p38, consisting of both active and inactive kinase, did not differ between groups. Representative blots are shown in the upper inset. ${ }^{\star} P<0.05,{ }^{\dagger} P<0.01$ vs control; ${ }^{\ddagger} P<0.05$ vs DS. 
a
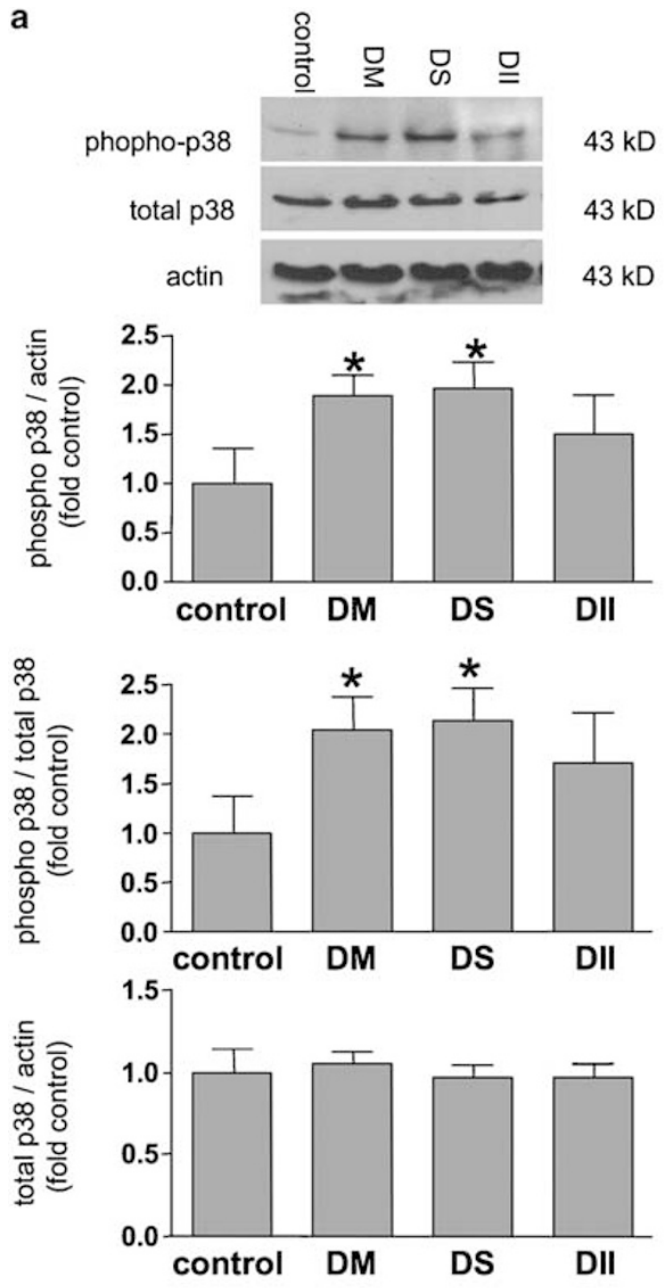

b

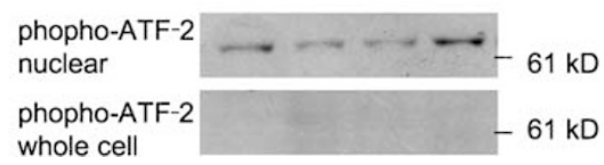

Figure 3 Renal cortical expression of phosphorylated (P-p38) and total p38 MAPK in nuclear fractions. Western blot analysis was used to determine p38 expressions, as described in Materials and methods (a). Nuclear expression of P-p38 (P-p38/actin) and P-p38/total p38 protein ratios were increased in moderately hyperglycemic (DM) and severely diabetic rats (DS), but not in diabetic rats treated with intensive insulin (DII), as compared with control animals. There were no differences in total nuclear p38 between the groups. Representative blots are shown in the upper inset. ${ }^{\star} P<0.05$ vs control. (b) Enrichment of nuclear fractions with the phospho-ATF-2 in control (left two lanes) and diabetic rats (right two lanes). Equal amounts $(60 \mu \mathrm{g})$ of nuclear extracts and whole-cell nuclei-free homogenates were analyzed by Western blocking under identical conditions using a primary antibody against phospho-ATF-2 (Thr 69/71). Unlike the whole-cell nucleifree homogenates, nuclear fractions revealed $70 \mathrm{kDa}$ band corresponding to endogenous phospho-ATF-2.

P-p38 was present in diabetic animals. Glomerular epithelial cells and parietal cells of Bowman capsule were the most frequent P-p38-positive glomerular cell types in diabetic rats, and occasionally in control animals (Figure 4j).
We next tested for the activation state of the p38 activators MKK3 and MKK6. There were no differences in phosphoMKK3/6 (the activated form), total MKK3, and phospho/ total MKK3/6 expression between the diabetic rats and controls (Figure 5). With respect to observed lack of differences in upstream MKK3/6 activity between control and diabetic rats, we further explored TAB1, as a possible modulator of p38 activity in the diabetic kidney. As demonstrated in Figure 6, TAB1 expression paralleled markers of p38 activity with marked increases in DM and DS rats $(P<0.001)$, and no statistically significant change in DII.

Because activation of p38 reflects a dynamic equilibrium between phosphorylation and dephosphorylation, downregulation of p38-directed phosphatase activity was considered. Unexpectedly, these measurements revealed increases in MKP-1 expression in all groups of diabetic rats irrespective of level of metabolic control and insulin treatment (Figure 7).

\section{Renal p38 Activity in Diabetic Rats with Established Nephropathy}

As shown in Table 2, 12-month-old diabetic rats demonstrated reduced weight gain, renal hypertrophy, increases in BG concentration, and HBA1c, and markedly elevated albuminuria, as compared with age-matched controls. The latter parameter corresponded to substantial glomerulosclerosis observed in DM12 (Figure 8b). Both p38 kinase activity assay (Figure 9) and determination expression of P-p38 (Figure 10) revealed marked increases in DM12 compared with controls. Immunohistochemical localization of active p38 showed most intensive stain found in macula densa cells and in distal tubules (Figure $8 \mathrm{c}$ and e).

\section{DISCUSSION}

Using several independent indices of p38 kinase activity, severely and moderately diabetic rats demonstrated increases in renal cortical p38 MAPK activity, as compared with agematched non-diabetic animals, albeit the P-p38/total ratio in DM was lower than in DS. p38 activity and P-p38 expression in diabetic animals were attenuated by intensive insulin treatment and improved metabolic control with most of the parameters characterizing p38 activity not statistically different from controls. Analysis of nuclear p38 expression showed the same pattern as in nuclei-free whole-cell fractions, suggesting that the process of nuclear translocation or nuclear activation of the kinase is not significantly altered or modulated by hyperglycemia or insulin. The absence of group differences in total p38 protein expression indicates that the diabetes-induced changes in p38 signaling pathway occur at the posttranslational level.

Previous in vitro studies have demonstrated activation of p38 MAPK in vascular and renal cells incubated in highglucose media, ${ }^{11-16,21}$ and in isolated glomeruli harvested from STZ-diabetic rats without insulin treatment. ${ }^{2,15,22}$ In addition, major metabolic consequences of hyperglycemia, such as activation of the hexosamine pathway and accumu- 

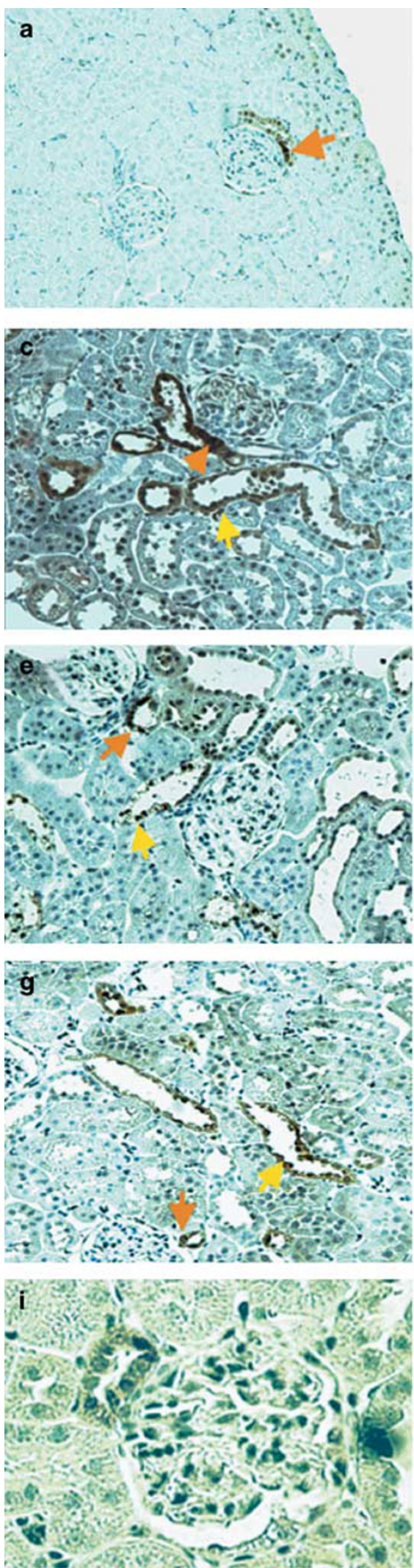
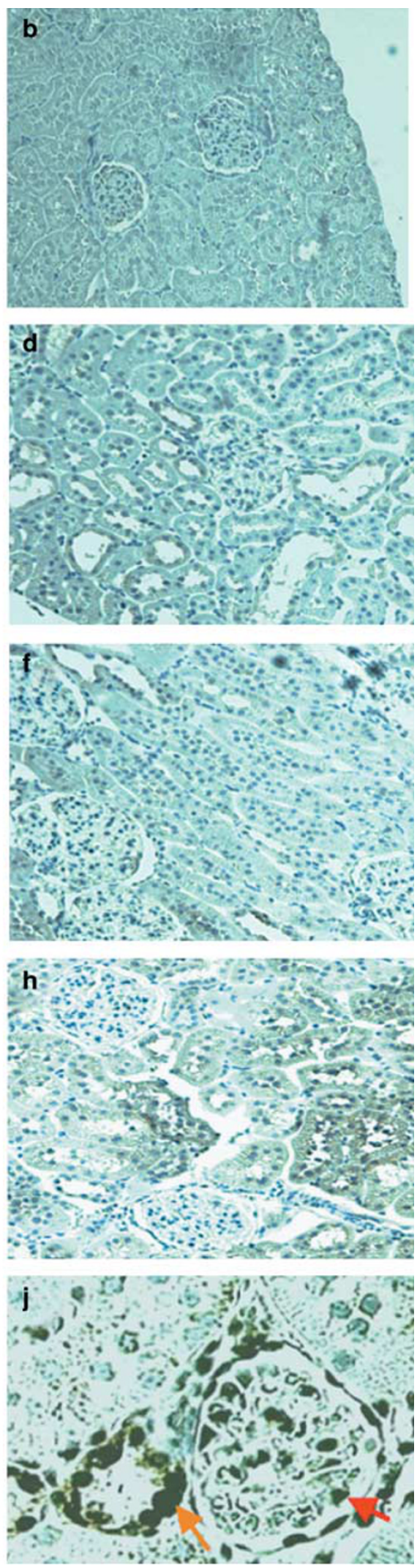

Figure 4 Immunohistochemical localization of active p38. In all groups of rats, phospho-p38 was predominantly localized in macula densa cells (orange arrows). Unlike the control animals (a, b), moderately diabetic $(\mathbf{c}, \mathbf{d})$, severely diabetic $(\mathbf{e}, \mathbf{f})$, and intensively treated diabetic rats $(\mathbf{g}, \mathbf{h})$ also demonstrated phospho-p38 immunoreactivity in distal tubules (yellow arrows). Panels $\mathbf{b}, \mathbf{d}, \mathbf{f}$, and $\mathbf{h}$ show control adjacent sections incubated with non-immune IgG. Lower panels show higher power glomerular images with adjacent macula densa in the control (i) and severely diabetic rat (j). These images show occasional phospho-p38immunoreactive glomerular cells, comprising mainly of podocytes (red arrow) and parietal cells of the Bowman capsule of diabetic rats. Sections of each diabetic kidney were processed in parallel with appropriate control tissue. Nephron segments were identified by characteristic palisade arrangements of cells (macula densa), and by the lack of brush border, larger lumina, and higher nuclear density (distal tubule). 


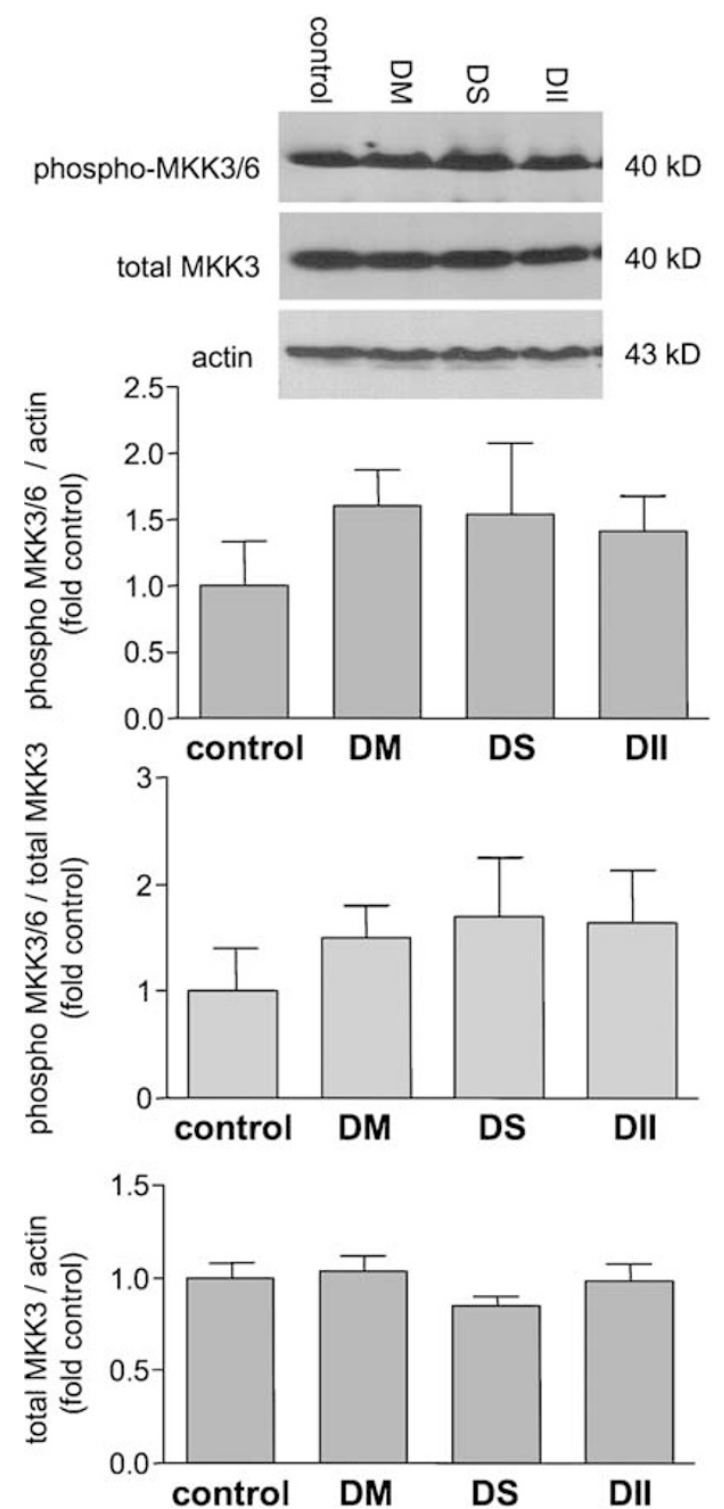

Figure 5 Renal cortical protein expression of phosphorylated MKK3/6 (P-MKK3/6) and total MKK3. Western blot analysis was used to determine MKK3/6 protein expressions, as described in Materials and methods. There were no differences in P-MKK3/6 (P-MKK3/6/actin), P-MKK3/6/total MKK3 ratios, and total MKK3 (total MKK3/actin) between moderately and severely hyperglycemic diabetic rats (DM and DS), intensively treated diabetic rats (DII), and controls. Representative blots are shown in the upper inset. ${ }^{*} P<0.05$ vs other groups of rats.
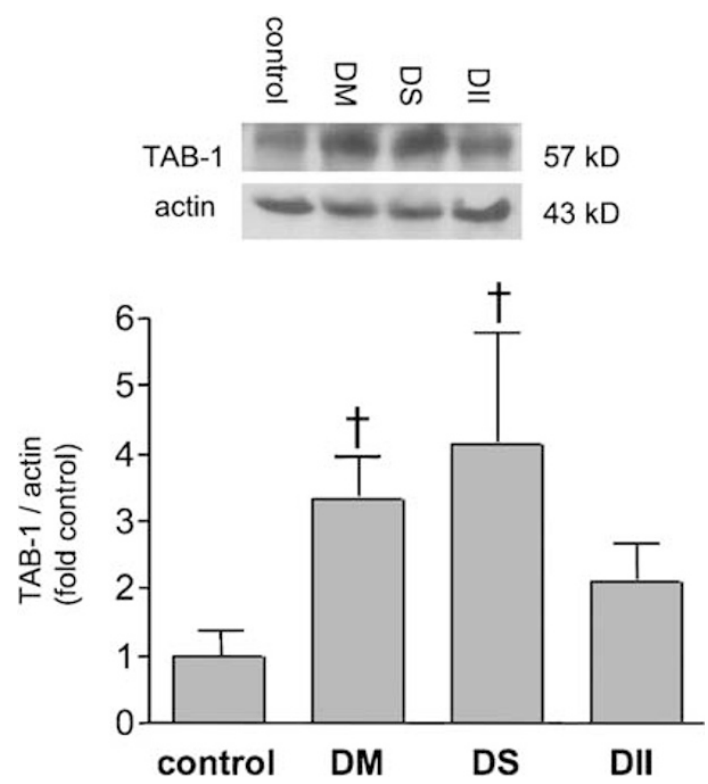

Figure 6 TAB1 expression in control and diabetic rats. Renal cortical TAB1 protein expression, determined by Western blotting, was significantly increased in moderately diabetic rats (DM), and in rats with severe diabetes (DS), as compared to control animals. Representative blots are shown in the upper inset. ${ }^{*} P<0.05,{ }^{\dagger} P<0.01$ vs control.

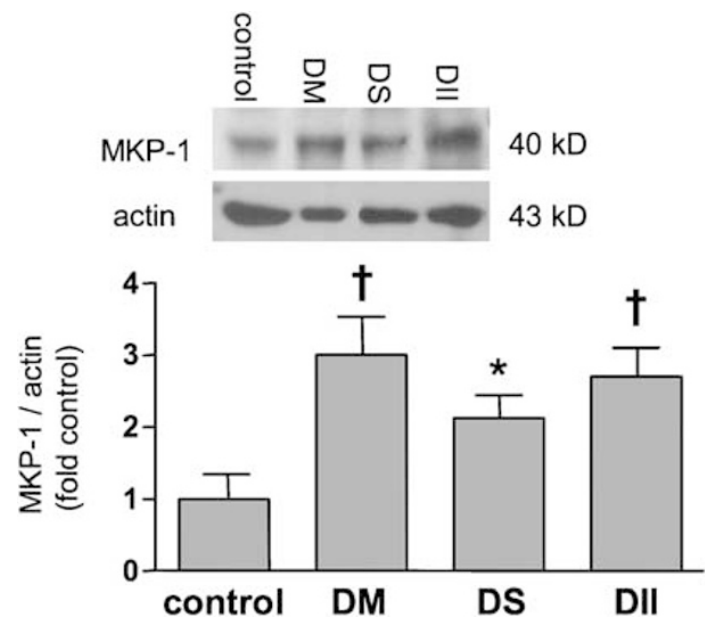

Figure 7 MKP-1 expression in control and diabetic rats. MKP-1 protein expression was determined by Western blotting, as described in Materials and methods. All groups of diabetic controls, irrespective of metabolic control and insulin treatment, demonstrated increased MKP-1 expression as compared with control animals. Representative blots are shown in the upper inset. Abbreviations as in Figure 6. ${ }^{\star} P<0.05,{ }^{\dagger} P<0.01$ vs control.

Table 2 General characteristics in control and diabetic rats after 12 months of follow-up

\begin{tabular}{|c|c|c|c|c|c|c|c|}
\hline Group & $n$ & BWT (g) & LKW (g) & LKW/BWT & $\mathrm{BG}(\mathrm{mg} / \mathrm{dl})$ & HBA1c (\%) & UalbV (mg/24h) \\
\hline C12 & 4 & $586 \pm 16$ & $1.72 \pm 0.08$ & $0.29 \pm 0.02$ & $83 \pm 8$ & $3.6 \pm 0.9$ & $33.3 \pm 12.5$ \\
\hline
\end{tabular}

BWT, body weight; LKW, left kidney weight; BG, blood glucose; HBA1c, glycosylated hemoglobin; UalbV, urinary albumin excretion; C12, age-matched control rats; DM12, moderately hyperglycemic diabetic rats after 12 months of diabetes.

${ }^{\mathrm{a}} \mathrm{P}<0.05$ vs $\mathrm{C} 12 ;{ }^{\mathrm{b}} \mathrm{P}<0.01$ vs $\mathrm{C} 12$. 

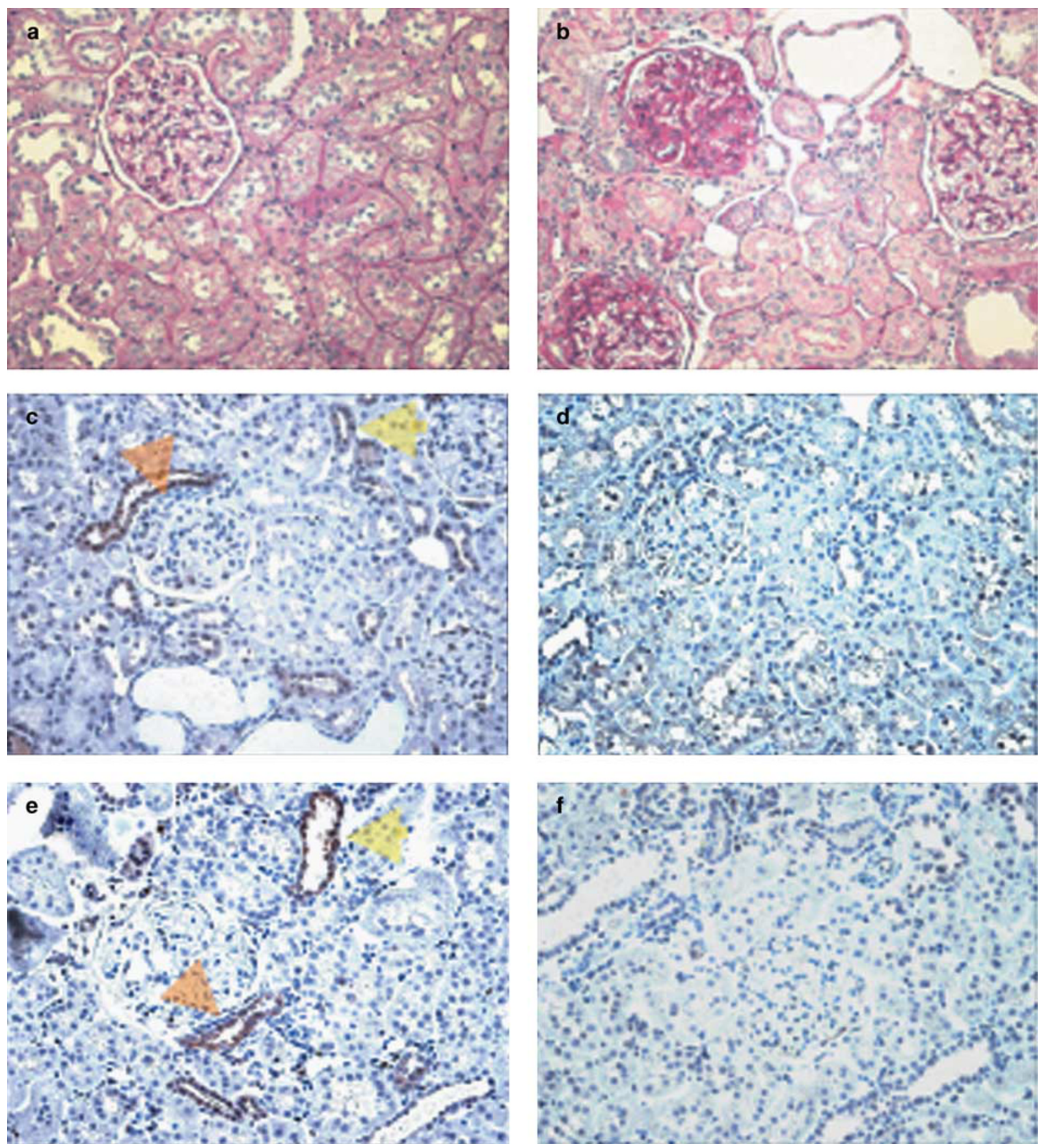

Figure 8 Glomerular changes and renal cortical localization of phosphorylated p38 (P-p38) in diabetic rats after 12 months of diabetes and in age-matched control animals. Unlike the age-matched controls (a), 12-month diabetic rats developed marked glomerulosclerosis (b). P-p38 immunoreactivity was apparent in macula densa cells (orange arrows) and distal tubules (yellow arrows) both in control (c) and diabetic animals (e). Notably, no phosphop38-positive cells were detectable in glomeruli. Panels $\mathbf{d}$ and $\mathbf{f}$ show adjacent sections incubated with non-immune IgG.

lation of products of glycation reactions, and oxidative stress, have been shown to induce p38 signaling in mesangial cells, podocytes and in proximal tubular cells in vitro. ${ }^{3,16,23-25}$ In agreement with these findings, present studies, conducted in more clinically relevant settings, show that poor metabolic control is the major determinant of p38 activity in the diabetic kidney. Our findings are also in agreement with limited in vivo evidence in the setting of Type 1 diabetes, suggesting increased expression of P-p38 protein expression in wholekidney homogenates harvested from mice early after induction of STZ-diabetes. ${ }^{17}$

At early stages of experimental nephropathy, active p38 has been previously localized in glomeruli, in particular in podocytes and mesangial cells, ${ }^{2,15,17,22}$ and in distal tubules. ${ }^{17}$ In 


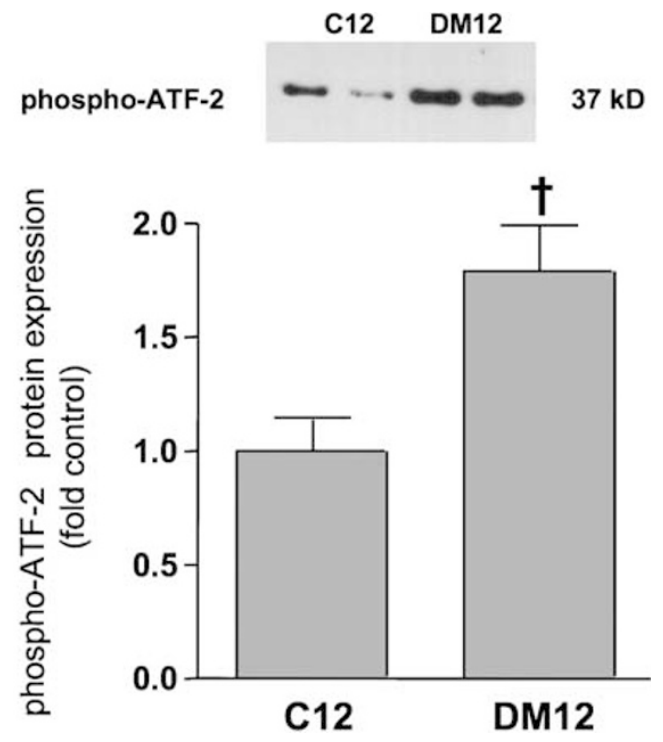

Figure 9 Renal cortical p38 MAPK activity in rats after 12 months of diabetes (DM12) and age-matched non-diabetic control animals (C12). Renal cortical p38 activity was determined by in vitro kinase assay using ATF-2 as a substrate. DM12 demonstrated increased renal cortical p38 activity as compared with $\mathrm{C} 12$. A representative blot of phospho-ATF-2 is shown in the upper panel. ${ }^{\dagger} P<0.01$ vs $\mathrm{C} 12$.

addition to these localizations, present immunohistochemical findings show that macula densa and adjacent portions of the nephron are the major sites of active p38 expression in the diabetic kidney. As discussed below, this finding may have functional and pathophysiological consequences.

In previous studies, increases in renal p38 activity in experimental diabetes were paralleled by increased protein and mRNA expression of its upstream kinase MKK3/6. ${ }^{2}$ In contrast, active MKK3/6 expression in diabetic rats in the present study was not significantly higher than in controls. This lack of clear parallel increase in activation of upstream MKK3/6 with p38 suggests possible involvement of other signaling molecules.

TAB1 is a scaffold protein that binds components of transforming growth factor- $\beta$ signaling cascade, including p38, leading to autophosphorylation and activation of the kinase. ${ }^{8}$ Several reports have implicated this protein in MKK3/6-independent p38 activation in experimental models of myocardial ischemia. ${ }^{26,27}$ To our knowledge, TAB1 has not been so far investigated in the kidney. Considering the wellestablished involvement of TGF- $\beta$ signaling cascade in the development of diabetic nephropathy, we hypothesized that TAB1 could play a role in p38 activation. Supporting this notion, expression of this protein paralleled p 38 activation in DM and DS, with a significant attenuation in animals with intensive insulin treatment.

As previously reported, hyperglycemia led to an increase in MKP-1 mRNA expression in mesangial cells in vitro, and in isolated glomeruli from STZ-diabetic rats. ${ }^{2,13}$ MKP-1 mRNA expression paralleled p38 activity including a decrease after
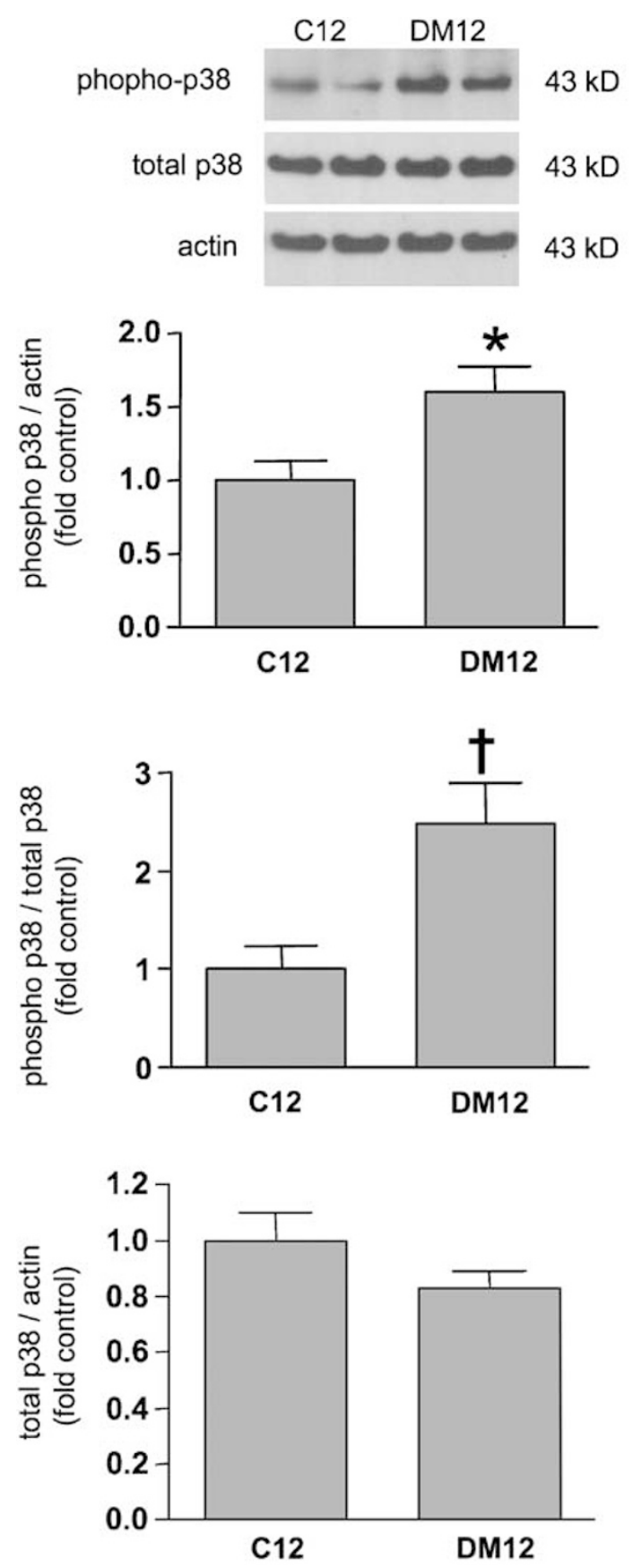

Figure 10 Renal cortical expression of phosphorylated (P-p38) and total p38 MAPK in rats after 12 months of diabetes (DM12) and age-matched non-diabetic control animals (C12). Western blot analysis was used to determine $\mathrm{p} 38$ expressions. In DM12, P-p38 expression (P-p38/actin) and Pp38/total p38 protein ratios were higher as compared with C12. Total p38 expression was not different between the groups. Representative blots are shown in the upper inset. ${ }^{\star} P<0.05,{ }^{\dagger} P<0.01$ vs $\mathrm{C} 12$.

4 months of diabetes. The authors postulated the existence of an MKP-1-mediated negative-feedback loop to dephosphorylate activated MAP kinase in diabetic glomeruli. Our observations of parallel activation of both p38 and MKP-1 in vivo are in agreement with this view. Moreover, MKP-1 may 
be also modulated by insulin..$^{28}$ Our observations in DII indicate that long-term exposure to high doses if insulin combined with mild hyperglycemia leads to higher MKP-1 expression.

Hyperglycemia or other components of diabetic milieu are not the only possible factors contributing to renal p38 activation in diabetes. Insulin per se activates p38 MAPK in the VSMC $^{29}$ and other cell types. ${ }^{30-32}$ This suggests that interactions between hyperglycemia, insulin levels, and the p38 pathway activity may be more complex. This issue is even more relevant with respect to the fact that previous experimental in vivo studies were performed in diabetic animals without insulin treatment. ${ }^{2,15,17}$ In diabetic rats, p38 activity was attenuated in parallel with improvement of glycemic control despite increasing doses of exogenous insulin. This information suggests that insulin per se does not play a significant role in activation of $\mathrm{p} 38$ signaling in diabetic renal cortex.

Specific activation of p38 in macula densa cells, as observed in the present studies, could be related to the composition of tubular fluid. Cheng et al. ${ }^{33}$ showed that $\mathrm{p} 38$ in macula densa cells is involved in regulation of cyclooxygenase-2 (COX-2) under certain conditions, such as low chloride tubular content. Since COX-2-derived metabolites have been implicated in the pathophysiology of progressive renal disease, including diabetes, ${ }^{34,35}$ this is an attractive pathway that may link p38 with the development of nephropathy in diabetes. Interestingly, p38 localization in diabetic rats was almost identical to that of COX-2, as observed in our previous studies. ${ }^{19}$ The link between p38 signaling and prostanoid production in VSMC and renal cells has been well established in vitro. ${ }^{11,36}$

Several reports have suggested that the time factor may be important in assessing the role of p38 in diabetic renal pathophysiology. ${ }^{2,15}$ Increases in $\mathrm{p} 38$ activity were observed in isolated glomeruli from STZ-diabetic rats at early stages of nephropathy with a decrease approximately 3 months after induction in diabetes. ${ }^{2,15}$ To address these issues, p38 activity and expression were further explored in diabetic rats after 12 months of duration of the disease with established renal morphological changes and significant albuminuria. Similar to early stages of nephropathy, these rats demonstrated marked increases in cortical p38 activity and expression of active kinase as compared with age-matched controls, with macula densa and distal tubules being the major sites of p38 immunoreactivity. Thus, these observations indicate that $\mathrm{p} 38$ activation is also a feature of established nephropathy. It should be noted that these immunohistochemical findings are not in disagreement with previously mentioned reports. ${ }^{2,15}$ In fact, we observed very weak glomerular phospho-p38 immunoreactivity in rats with long-standing diabetes. The observed increases in renal cortical p38 activity and expression are attributable to the large tubular pool of the kinase. This notion is supported by the available immunohistochemical findings in established human Type 2 diabetic nephropathy ${ }^{17,18}$ and in STZ-diabetic mice 8 months after induction of diabetes, ${ }^{17}$ suggesting a substantial tubular pool of active kinase.

Considering signaling events downstream of p38, activation of this cascade could play a role in a variety of mechanisms relevant for the development of diabetic nephropathy. These mechanisms could include deleterious effects such as activation of the renin-angiotensin system, ${ }^{14,23,37}$ oxidative stress, ${ }^{37}$ extracellular matrix (ECM) production and cellular hypertrophy, ${ }^{2}$ induction of growth cytokines, ${ }^{2,3}$ mesangial and podocyte apoptosis, ${ }^{16,24}$ and glucose-induced 12-lipooxygenase pathway signaling, ${ }^{22}$ as well as possibly protective effects. such as cytoskeleton reorganization in podocytes that temporarily prevents the development of albuminuria. ${ }^{15}$

Further insight into the pathophysiological significance of renal p38 activation in diabetes should be provided by longterm studies with p38 inhibitors in appropriate models. Although the long-term effects of p38 inhibition on the development of diabetic nephropathy have not been elucidated, study have shown that this intervention may attenuate the development of renal glomerular and interstitial lesions in non-diabetic models of kidney disease. ${ }^{38,39}$ In an experimental model of diabetic cardiomyopathy, chronic p38 inhibition attenuated the left ventricular dysfunction and prevented cardiac inflammation. ${ }^{40}$

In summary, renal cortical p38 MAPK activity and expression was increased in diabetic rats at early stages of nephropathy as well as in animals with advanced disease, as compared with age-matched non-diabetic animals. These diabetes-induced increases in $\mathrm{p} 38$ activity were attenuated by insulin treatment and the associated improvement in glycemic control. p38 activation in diabetes is likely to occur via multiple pathways involving scaffolding protein TAB1, and cannot be explained by downregulation of MKP-1.

\section{ACKNOWLEDGEMENT}

These studies were supported, in part, by grants to Dr Anderson from the Juvenile Diabetes Research Foundation (3-2001-851) and the NIH (DK 063231); to Dr Komers, by Grant \# MZO-00023001 provided by the Ministry of Healthcare of the Czech Republic; and to Dr Cohen (NIH grant DK-52494 and the Department of Veterans Affairs).

1. Kyriakis JM, Avruch J. Mammalian mitogen-activated protein kinase signal transduction pathways activated by stress and inflammation. Physiol Rev 2001;81:807-869.

2. Kang SW, Adler SG, Lapage J, et al. p38 MAPK and MAPK kinase $3 / 6$ mRNA and activities are increased in early diabetic glomeruli. Kidney Int 2001;60:543-552.

3. Burt DJ, Gruden G, Thomas SM, et al. P38 mitogen-activated protein kinase mediates hexosamine-induced TGFbeta1 mRNA expression in human mesangial cells. Diabetologia 2003;46:531-537.

4. Force T, Bonventre JV. Growth factors and mitogen-activated protein kinases. Hypertension 1998;31:152-161.

5. Tian W, Zhang Z, Cohen DM. MAPK signaling and the kidney. Am J Physiol 2000;279:F593-F604.

6. Ushio-Fukai $M$, Alexander RW, Akers $M$, et al. p38 mitogen-activated protein kinase is a critical component of the redox-sensitive signaling pathways activated by angiotensin II. J Biol Chem 1998;273:1502215029. 
7. Muller E, Burger-Kentischer A, Neuhofer W, et al. Possible involvement of heat shock protein 25 in the angiotensin II-induced glomerular mesangial cell contraction via p38 MAP kinase. J Cell Physiol 1999;181:462-469.

8. Ge B, Gram H, Di Padova F, et al. MAPKK-independent activation of p38alpha mediated by TAB1-dependent autophosphorylation of p38alpha. Science 2002;295:1291-1294.

9. Keyse SM. Protein phosphatases and the regulation of mitogenactivated protein kinase signalling. Curr Opin Cell Biol 2000;12:186192.

10. Franklin CC, Kraft AS. Conditional expression of the mitogen-activated protein kinase (MAPK) phosphatase MKP-1 preferentially inhibits p38 MAPK and stress-activated protein kinase in U937 cells. J Biol Chem 1997;272:16917-16923.

11. Igarashi $M$, Wakasaki $H$, Takahara $N$, et al. Glucose or diabetes activates p38 mitogen-activated protein kinase via different pathways. J Clin Invest 1999;103:185-195.

12. Natarajan $\mathrm{R}$, Scott $\mathrm{S}$, Bai $\mathrm{W}$, et al. Angiotensin II signaling in vascular smooth muscle cells under high glucose conditions. Hypertension 1999;33:378-384.

13. Kang MJ, Wu X, Ly H, et al. Effect of glucose on stress-activated protein kinase activity in mesangial cells and diabetic glomeruli. Kidney Int 1999;55:2203-2214.

14. Zhang SL, Tang SS, Chen X, et al. High levels of glucose stimulate angiotensinogen gene expression via the P38 mitogen-activated protein kinase pathway in rat kidney proximal tubular cells. Endocrinology 2000;141:4637-4646.

15. Dai T, Natarajan R, Nast CC, et al. Glucose and diabetes: effects on podocyte and glomerular p38MAPK, heat shock protein 25, and actin cytoskeleton. Kidney Int 2006;69:806-814.

16. Susztak K, Raff AC, Schiffer M, et al. Glucose-induced reactive oxygen species cause apoptosis of podocytes and podocyte depletion at the onset of diabetic nephropathy. Diabetes 2006;55:225-233.

17. Adhikary L, Chow F, Nikolic-Paterson DJ, et al. Abnormal p38 mitogenactivated protein kinase signalling in human and experimental diabetic nephropathy. Diabetologia 2004;47:1210-1222.

18. Sakai N, Wada T, Furuichi K, et al. Involvement of extracellular signalregulated kinase and p38 in human diabetic nephropathy. Am J Kidney Dis 2005;45:54-65.

19. Komers R, Lindsley JN, Oyama TT, et al. Immunohistochemical and functional correlations of renal cyclooxygenase-2 in experimental diabetes. J Clin Invest 2001;107:889-898.

20. Widmann C, Gibson S, Jarpe MB, et al. Mitogen-activated protein kinase: conservation of a three-kinase module from yeast to human. Physiol Rev 1999;79:143-180.

21. Wilmer WA, Dixon CL, Hebert C. Chronic exposure of human mesangial cells to high glucose environments activates the p38 MAPK pathway. Kidney Int 2001;60:858-871.

22. Kang SW, Natarajan R, Shahed A, et al. Role of 12-lipoxygenase in the stimulation of p38 mitogen-activated protein kinase and collagen alpha5(IV) in experimental diabetic nephropathy and in glucosestimulated podocytes. J Am Soc Nephrol 2003;14:3178-3187.

23. Hsieh TJ, Fustier $\mathrm{P}$, Zhang SL, et al. High glucose stimulates angiotensinogen gene expression and cell hypertrophy via activation of the hexosamine biosynthesis pathway in rat kidney proximal tubular cells. Endocrinology 2003;144:4338-4349.

24. Liu BF, Miyata S, Hirota Y, et al. Methylglyoxal induces apoptosis through activation of p38 mitogen-activated protein kinase in rat mesangial cells. Kidney Int 2003;63:947-957.
25. Li JH, Huang XR, Zhu HJ, et al. Advanced glycation end products activate Smad signaling via TGF-beta-dependent and independent mechanisms: implications for diabetic renal and vascular disease. FASEB J 2004;18:176-178.

26. Tanno M, Bassi R, Gorog DA, et al. Diverse mechanisms of myocardial p38 mitogen-activated protein kinase activation: evidence for MKKindependent activation by a TAB1-associated mechanism contributing to injury during myocardial ischemia. Circ Res 2003;93:254-261.

27. Li J, Miller EJ, Ninomiya-Tsuji J, et al. AMP-activated protein kinase activates p38 mitogen-activated protein kinase by increasing recruitment of p38 MAPK to TAB1 in the ischemic heart. Circ Res 2005;97:872-879.

28. Begum N, Ragolia L. High glucose and insulin inhibit VSMC MKP-1 expression by blocking iNOS via p38 MAPK activation. Am J Physiol 2000;278:C81-C91.

29. Igarashi $M$, Yamaguchi $H$, Hirata $A$, et al. Insulin activates p38 mitogenactivated protein (MAP) kinase via a MAP kinase kinase (MKK) 3/MKK 6 pathway in vascular smooth muscle cells. Eur J Clin Invest 2000;30:668-677.

30. Somwar R, Perreault M, Kapur $\mathrm{S}$, et al. Activation of p38 mitogenactivated protein kinase alpha and beta by insulin and contraction in rat skeletal muscle: potential role in the stimulation of glucose transport. Diabetes 2000;49:1794-1800.

31. Sweeney G, Somwar R, Ramlal T, et al. An inhibitor of p38 mitogenactivated protein kinase prevents insulin-stimulated glucose transport but not glucose transporter translocation in 3T3-L1 adipocytes and L6 myotubes. J Biol Chem 1999;274:10071-10078.

32. Keeton $A B$, Amsler MO, Venable DY, et al. Insulin signal transduction pathways and insulin-induced gene expression. J Biol Chem 2002;277:48565-48573.

33. Cheng $\mathrm{H}-\mathrm{F}$, Wang $\mathrm{J}-\mathrm{L}$, Zhang $\mathrm{M}-\mathrm{Z}$, et al. Role of p38 in the regulation of renal cortical cyclooxygenase- 2 expression by extracellular chloride. J Clin Invest 2000;106:681-688.

34. Wang J-L, Cheng H-F, Shappell S, et al. Cyclooxygenase-2 (COX-2) inhibition decreases proteinuria in a hypertensive diabetic model. J Amer Soc Nephrol 1999;10:A3505 (Abstract).

35. Cheng HF, Wang CJ, Moeckel GW, et al. Cyclooxygenase-2 inhibitor blocks expression of mediators of renal injury in a model of diabetes and hypertension. Kidney Int 2002;62:929-939.

36. Martineau LC, McVeigh LI, Jasmin BJ, et al. p38 MAP kinase mediates mechanically induced COX-2 and PG EP4 receptor expression in podocytes: implications for the actin cytoskeleton. Am J Physiol Renal Physiol 2004;286:F693-F701.

37. Hsieh TJ, Zhang SL, Filep JG, et al. High glucose stimulates angiotensinogen gene expression via reactive oxygen species generation in rat kidney proximal tubular cells. Endocrinology 2002;143:2975-2985.

38. de Borst MH, Navis G, de Boer RA, et al. Specific MAP-kinase blockade protects against renal damage in homozygous TGR(mRen2)27 rats. Lab Invest 2003;83:1761-1770.

39. Prakash J, Sandovici M, Saluja V, et al. Intracellular delivery of the p38 mitogen-activated protein kinase Inhibitor SB202190 [4-(4Fluorophenyl)-2-(4-hydroxyphenyl)-5-(4-pyridyl) $1 \mathrm{H}$-imidazole] in renal tubular cells: a novel strategy to treat renal fibrosis. J Pharmacol Exp Ther 2006;319:8-19.

40. Westermann D, Rutschow S, Van Linthout S, et al. Inhibition of p38 mitogen-activated protein kinase attenuates left ventricular dysfunction by mediating pro-inflammatory cardiac cytokine levels in a mouse model of diabetes mellitus. Diabetologia 2006;49:2507-2513. 